Questions vives

\section{Questions Vives}

Recherches en éducation

$N^{\circ} 29$ | 2018

La bienveillance en éducation : approches

compréhensives et critiques

\title{
La bienveillance en éducation au prisme de la notion de caring : pour un renouveau des pédagogies actives?
}

Benevolence in education through the prism of the notion of caring: for a renewal of active pedagogies?

\section{Thierry Piot}

\section{(2) OpenEdition}

Journals

\section{Édition électronique}

URL : https://journals.openedition.org/questionsvives/3556

DOI : 10.4000/questionsvives.3556

ISSN : 1775-433X

Éditeur

Université Aix-Marseille (AMU)

Édition imprimée

ISBN : 978-2-912643-53-7

ISSN : 1635-4079

Référence électronique

Thierry Piot, «La bienveillance en éducation au prisme de la notion de caring : pour un renouveau des pédagogies actives? », Questions Vives [En ligne], № 29 | 2018, mis en ligne le 19 décembre 2018, consulté le 21 septembre 2021. URL : http://journals.openedition.org/questionsvives/3556 ; DOI : https://doi.org/10.4000/questionsvives.3556

Ce document a été généré automatiquement le 21 septembre 2021.

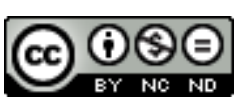

Questions Vives est mis à disposition selon les termes de la licence Creative Commons Attribution Pas d'Utilisation Commerciale - Pas de Modification 4.0 International. 


\title{
La bienveillance en éducation au prisme de la notion de caring : pour un renouveau des pédagogies actives?
}

\author{
Benevolence in education through the prism of the notion of caring: for a \\ renewal of active pedagogies?
}

Thierry Piot

\section{Introduction}

1 La Loi pour la refondation de l'école de $2013^{1}$ pose comme centrale une notion émergente dans le champ éducatif et scolaire: la bienveillance. Cette notion est mobilisée dans le texte de la loi comme dans la circulaire de rentrée $2014^{2}$ mais est marquée par une forme de flou conceptuel avec une valeur morale implicite, qui s'appuierait sans l'expliciter sur la dimension vocationnelle du métier d'enseignant-e.

2 Notre propos vise à discuter la notion de bienveillance d'un point de vue épistémologique et pragmatique en proposant une articulation avec la notion de care, qui lui est proche et qui a fait l'objet de recherches importantes dans le champ de la philosophie morale et politique aux USA depuis les années 1980, à partir notamment des travaux fondateurs de Carol Gilligan (1982).

Dans un premier temps, nous tenterons de rendre compte et de discuter cette notion de care/caring du point de vue nord-américain, en nous appuyant principalement sur les travaux de Joan Tronto (2009), qui apportent une consistance épistémologique à la notion de bienveillance, en proposant une lecture à la fois philosophique, sociale et politique.

Dans un deuxième temps, nous examinerons la manière dont le champ des soins adressés aux personnes les plus vulnérables s'est emparé de la notion de care, tant sur le plan 
conceptuel que pratique, en proposant de passer d'une logique de hiérarchisation à une logique de complémentarité avec la notion de cure.

À partir de cet éclairage, nous tenterons de discuter le concept de bienveillance dans le champ éducatif, en nous appuyant entre autres sur les travaux de Nel Noddings (1984), qui propose un nouvel éclairage pour les postures pédagogiques déjà défendues par les pédagogies actives, mais souvent en dehors de l'institution scolaire, et met l'accent sur des points de tension souvent restés dans l'ombre, pour aborder la question de la prise en charge des difficultés scolaires.

6 La bienveillance est plutôt un concept de sens commun, peu élucidé sur le plan épistémologique. Elle entretient une forte convergence de signification avec la notion de care, elle-même difficile à traduire en français, comme le note Eliane Rothier Bautzer (2016). Ces deux termes ont pour origine « un souci de l'autre ». La bienveillance à l'égard d'autrui se caractérise plus comme une disposition, proche de l'empathie, qui vise non seulement à le protéger mais aussi à favoriser son épanouissement et à rechercher son bien-être, avec l'idée d'une posture morale associée, tandis que le care est à rapprocher de la sollicitude envers autrui, c'est-à-dire l'idée de lui venir en aide, d'en prendre soin, notamment si cet autrui est dans une situation vulnérable qui entrave son pouvoir d'agir.

\section{Le care : une notion problématisée par les travaux féministes nord-américains dans les années 1980}

\subsection{Une pensée alternative et un projet politique}

7 Dès l'aube des années 1980 aux USA, des travaux vont développer un courant de pensée critique et alternatif aux courants classiques, autour de la notion de care. S'y rattachant directement, les travaux sur la notion d'émancipation et d'empowerment (Solomon, 1976) qui touchent de nombreux secteurs de la société (intervention sociale, soins, éducation, développements communautaires, droits des outsiders que sont les femmes, les minorités noires ou gays et lesbiennes), accordent du crédit à la parole des plus faibles, à l'expression des personnes vulnérables qui occupent des positions marginalisées et dominées (Wise, 2005). Une controverse forte existe à propos de la notion de justice, entre d'une part John Rawls (1971/1997), qui défend l'idée de justice adossée à des principes universels et atemporels, et d'autre part les tenants de l'éthique du care qui proposent de discuter la notion de justice à partir de l'expérience des personnes, ancrée dans une temporalité et un contexte socio-déterminés, incluant des interdépendances.

8 Joan Tronto s'engage dans les luttes politiques des années 1970 aux USA, luttes qui prennent de l'ampleur dans plusieurs universités américaines dans le sillage de la fin de la guerre du Vietnam, et proposent un regard différent sur le politique et l'éthique en politique, c'est-à-dire les principes de gouvernement et leur traduction dans la vie de la cité. Elle va centrer ses travaux sur la notion de care/caring ${ }^{3}$ et développer, au sein du mouvement auquel elle appartient, une pensée originale qui l'amène à discuter le care au croisement de trois registres d'une part distincts - pour plus de clarté analytique -, et d'autre part enchâssés et complémentaires - pour servir une visée synthétique et holiste : un registre éthique, central dans sa pensée, un registre politique et social ainsi qu'un registre pratique et instrumental (sur lequel elle s'appesantit moins). 
9 Le registre éthique s'ancre dans la nécessité de respecter autrui de manière juste et incarnée. Ce sont les travaux en "éthique du care», issus des courants féministes nordaméricains, qui, les premiers, ont cherché à conceptualiser cette notion ordinaire et profane. Ici, la dimension morale n'est pas seulement mue par des principes et des valeurs mais se met à l'épreuve des situations pratiques ordinaires qui participent à la définir. Mais, et Joan Tronto s'en défend longuement, ce n'est pas d'une morale « locale » créée par et pour un groupe dominé (ethno-centrée pour un groupe culturel particulier - ici les Noirs américains -, ou socio-centrée sur une catégorie sociale précarisée, ou genro-centrée - qui serait en l'occurrence propre aux femmes -, dont il est question, mais d'une morale à prétention générale. Elle critique implicitement la morale classique, héritée des philosophes européens, qu'elle juge trop impersonnelle. Elle vise à prendre en compte d'emblée la place et le sort réservés aux marginaux du système social et politique. Cette morale inclut une mise à l'épreuve de sa validité dans la vie ordinaire et dans l'expérience sensible de chacun, lesquelles méritent une attention de premier plan. Et, comme le souligne Noddings (2012), l'expérience sensible de chacun est à la base une expérience de la relation humaine. Sont considérés comme dignes d'intérêt des gestes, des attitudes, des interactions langagières ou non qui sont autant de « petites choses » qui apparaissent au premier abord anodines (Hesbeen, 2014) mais qui sont porteuses de significations pour les personnes vulnérables et dont la prise en compte attentive permet d'indiquer à la personne qui en bénéficie qu'elle existe en tant que sujet digne d'attention pour la personne qui en prend soin, au-delà des traits qui la stigmatisent. Joan Tronto est singulièrement critique lorsqu'elle estime qu'il faut envisager autrement le concept occidental de liberté qu'elle estime trop attaché aux notions d'autonomie et d'indépendance qui séparent les individus, alors que d'après elle, la vie des humains en général est possible aussi, et d'abord, par l'existence de réseaux d'interdépendance, de relations sociales intriquées et en même temps peu visibles.

Le registre politique et social est fortement en continuité avec le registre éthique. Joan Tronto analyse que les pratiques de prise en compte d'autrui, qui fabriquent de l'interdépendance et du lien social, éminemment nécessaires au fait que les hommes puissent vivre ensemble si possible harmonieusement, ou au moins avec des violences limitées et régulées, sont invisibilisées dans la société où dominent, dans l'arène politique et dans la vie de la cité, des discriminations et des segmentations préjudiciables à la cohésion de la société. Cette absence de visibilité du care/caring sur le devant de la scène politique et sociale nuit à la possibilité même de la mettre en mots, en controverse, de la penser pour changer l'ordre des choses, dans une logique d'émancipation. Cette invisibilité n'est pas un hasard: elle trouverait son origine dans le fait que les pratiques de care/caring (soins aux démunis, aux personnes âgées, aux personnes handicapées, aux plus faibles et aux plus vulnérables de manière générale) sont méprisées, mises à l'arrière-plan, mal connues, considérées presque comme subsidiaires. Les personnes qui sur le plan professionnel exercent ces métiers (aides-ménagers-ères, aides-soignant-e-s, aides médico-psychologiques, assistant-e-s d'éducation, voire éducateurs-trices, animateurs-trices, enseignant-e-s, infirmiers-ères, formateurs-trices pour adultes...) qui sont des métiers de services adressés à autrui, désignés également par l'expression "métiers de la relation", sont peu formées, peu diplômées et généralement peu rémunérées: l'activité de caring qui est au cœur de ces métiers est implicitement considérée comme d'essence maternelle, c'est-à-dire qu'elle serait exclusivement attachée à des dispositions ou aptitudes intrinsèques à la nature supposée des femmes, à 
une forme de vertu, qui ne s'apprennent que pas ou peu, car proches d'activités quotidiennes et domestiques, très genrées dans une société dominée par les hommes. De fait, les personnes qui exercent ces métiers appartiennent à des catégories dominées : femmes, immigrés... Le caring, de ce fait, est peu regardé comme une activité contributive au bien-vivre ensemble et moins encore comme participant de la création de richesses. Il est à la périphérie et c'est justement ce que l'analyse diagnostique de Joan Tronto s'efforce de dénoncer en proposant de prendre au sérieux les pratiques de caring, de les considérer d'emblée comme productrices d'épanouissement et de richesse.

11 À partir d'une analyse qui parfois manque de nuances, Joan Tronto esquisse un projet politique et social qui donnerait centralité, visibilité et reconnaissance aux fonctions d'interdépendance qui relient les humains dans leur vie. Le principe qui soutient la colonne vertébrale de ce projet est celui d'une justice équitable, qui se soucierait explicitement que soient pris en compte, avec sollicitude et bienveillance, les besoins de chaque personne (et pas seulement des personnes vulnérables, sauf à penser qu'on est tous vulnérables ou potentiellement vulnérables).

Le registre pratique et instrumental n'est pas déconnecté des registres précédents : il en traduit la mise en œuvre concrète. S'il est pensé pour lui-même, il risquerait d'être réifié, réduit à des débats techniques, normatifs, autour de dispositifs à mettre en place et dont on contrôle l'efficacité de manière externe. Ce registre pratique et instrumental s'incarne dans des pratiques de caring organisées autour des besoins d'autrui, d'abord dans le domaine de la vie quotidienne, besoins qu'il exprime directement de manière explicite ou qui requièrent une phase de dialogue pour être identifiés de manière pertinente. Les pratiques sont enchâssées et multi-scalaires, c'est-à-dire allant du soin à la personne ( micro), au soin vers la communauté de vie pour un territoire donné (meso) et au soin de la société (macro), visant une forme de cohérence globale. Tronto insiste pour qu'on ne retienne pas de son projet politique une dimension "palliative ", réservée aux seules personnes vulnérables qui seraient à catégoriser pour être prises en charge de manière spécifique. Son propos, précise-t-elle, a une dimension inclusive et générale.

Si la traduction du terme anglo-saxon care pose problème pour trouver un terme unique en français (attention à l'autre, sollicitude, bienveillance...), on peut s'appuyer sur la définition que Joan Tronto et Bérenice Fischer (1991) proposent, qui insiste sur le fait de quitter une forme de dépendance - ou d'assujettissement plus ou moins subreptice et intériorisé - avec justice et dignité, via la prise en compte de ses besoins pour préserver ou développer son pouvoir d'agir :

Au niveau le plus général, nous suggérons que le care soit considéré comme une activité générique qui comprend tout ce que nous faisons pour maintenir, perpétuer et réparer notre « monde ", de sorte que nous puissions y vivre aussi bien que possible. Ce monde comprend nos corps, nous-mêmes et notre environnement, tous éléments que nous cherchons à relier en un réseau complexe en soutien à la vie.

Du point de vue de Joan Tronto, ces propositions ne sont ni laxistes, ni romantiques, ni porteuses d'angélisme : il ne s'agit pas seulement de manifester une forme de gentillesse, de douceur ou d'adresser un sourire à une personne... ce qui est souvent considéré comme des marqueurs de la sollicitude, mais qui sont des marqueurs de surface, oublieux que la bienveillance est plus consistante et complexe que des signaux extérieurs, parfois trompeurs. Les pratiques de caring ne sont ni propres aux faibles, ni réservées aux dominés: c'est un véritable projet moral et politique alternatif, finalisé par une émancipation globale où l'interdépendance entre les personnes prend le pas sur la 
compétition dans l'arène politique et quotidienne. Il s'agit de transformer la morale classique ordonnée à des principes immuables et descendants, en l'irriguant par l'expérience quotidienne de chacun à la fois singulière et sensible, dans une logique ascendante, ordonnée à la recherche de justice et de dignité. Ainsi, une centralité inédite est accordée aux pratiques de care/caring ordinaire, centralité qui suppose une tension nouvelle entre autonomie de chaque sujet et interdépendance des sujets entre eux.

\subsection{Le care/caring, comme intention et comme processus à quatre temps} dans une situation donnée et à les identifier. Nous signalons ici l'importance stratégique de cette phase d'identification des besoins d'autrui, en indiquant une dérive : la personne qui prend soin, par habitude ou parfois par facilité, peut être tentée de considérer son propre avis comme suffisant et de contourner une phase de dialogue essentielle : associer autrui à cette étape en lui donnant la parole - au sens plein de cette expression - pour écouter comment elle formule ce qui est nécessaire. Et cela même si cette formulation est incomplète, maladroite, entravée par le fait que, justement, cette personne est en situation de vulnérabilité... mais en même temps possède un ressenti et une expertise pratique de sa situation qui n'est pas remplaçable. penser et d'organiser l'aide à apporter pour prendre en compte les besoins particuliers, 
dans un contexte donné, pluridimensionnel, à la fois sur le plan humain et relationnel mais aussi sur les plans matériel, social, temporel, spatial. Il s'agit, au sens de Dewey (1938/2006), de problématiser la prise en charge pour la rendre opérationnalisable. Cela renvoie à penser de manière recevable le rapport moyen-fin dans un environnement et une situation donnés : c'est, en complément de la phase précédente, un véritable travail d'ingénierie du care, puisque c'est une démarche de résolution de problème singulier dans un contexte contraint dont il est question, et pas seulement un geste automatique qui aurait une force d'évidence et d'automaticité pour celle ou celui qui aurait les dispositions naturelles ou les aptitudes culturellement intériorisées pour le réaliser. Cette notion d'ingénierie du care, qui serait un marqueur des métiers de service adressés à autrui, suggère de penser de manière renouvelée la notion de situation de travail, doublement centrée: d'une part sur l'objet de service dans sa dimension technique (les protocoles d'asepsie dans les soins infirmiers, la dimension didactique en enseignement...), et d'autre part en prenant en compte un second centre de gravité, le souci conscientisé d'autrui. Cela induit de combiner deux registres de compétences hétérogènes et complémentaires dans ces métiers : des compétences sur l'objet, plus rationnelles et objectivables, et des compétences relationnelles et communicationnelles, construites par expérience et par un travail réflexif, à la fois individuel et collectif (Piot, 2008).

La phase 3, care giving (prendre soin), consiste en la réalisation effective du caring. À la suite de la phase précédente, cela revient à mettre à l'épreuve du réel la solution construite. C'est la face visible de l'activité de caring, dans une double logique: (1) prendre soin avec bienveillance de la personne et (2) apporter une aide efficace en regard de l'objet de service dont il est question dans la situation considérée (aide à la personne, enseignement, santé, formation...). Cela demande de conduire une activité complexe, multi-registres, d'en contrôler le déroulement, d'en réguler le décours le cas échéant.

La phase 4, care receiving (recevoir le soin), est importante car elle concerne le message en retour du bénéficiaire (attitude, comportement, commentaires), la reconnaissance possible ressentie ou exprimée par autrui qui est bénéficiaire. C'est une forme de feedback, qui est aussi une forme de contre-don (Mauss, 1924/2013). Cette phase permet de connaître la manière dont autrui a reçu le caring qui lui a été adressé, à la fois comme service objectif et comme processus de reconnaissance et de soutien ajusté. Si le besoin est mal évalué à l'origine ou encore si le service rendu est défaillant de quelque manière, ou s'il induit des effets secondaires non désirés et imprévus, la parole d'autrui est un moyen - certes pas infaillible - de le repérer et, le cas échéant, d'ajuster l'aide en cours, de la corriger... surtout si elle s'inscrit dans une forme d'accompagnement au long d'un parcours, comme c'est souvent le cas dans les prises en charge : personnes en situation de dépendance, aide aux apprentissages scolaires, réinsertion sociale ou professionnelle... Donner la parole au bénéficiaire et l'écouter rompt avec certaines logiques classiques qui parfois s'imposent par une seule prise en charge instrumentale et n'évaluent pas ou peu leur impact, généralement agissant sur une temporalité longue, sur le plan relationnel et finalement sur le plan de la place symbolique accordée à la personne en tant que sujet, qui est au fondement de sa dignité.

Les travaux sur le care/caring ainsi que les débats auxquels ils donnent lieu aux USA vont trouver une audience croissante en France à partir des années 2000, présentés et discutés notamment par Sandra Laugier et Patricia Paperman (2006) ou Liane Mozère ${ }^{4}$. 


\section{Care/caring vs cure/curing : l'apport de la réflexion sur la bienveillance à accorder aux malades dans les métiers du soin}

Le care comme attention ciblée et bienveillante pour le mieux-être d'autrui et le caring comme pratique associée ne sont pas uniquement ce que Joan Tronto désigne comme la force des faibles (p. 168). Le couple care/caring renvoie à de nombreuses réflexions depuis une vingtaine d'années, réflexions qui s'écartent de l'analyse éthico-politique originelle de Tronto pour se déployer dans des analyses éthico-professionnelles. C'est notamment le cas de la santé où les modalités de prise en charge à l'hôpital, en établissement d'hébergement pour personnes âgées dépendantes (EHPAD) ou même à domicile se transforment avec, comme indicateurs possibles, les chartes de déontologie, les projets pour les patients, le droit aux patients, même si, comme le met en évidence Éliane Rothier Bautzer (2013) dans le titre d'un ouvrage, dans nombre de situations de soin - notamment le soin chronique - comme en formation des personnels soignants, le care (reste) négligé.

Cette place périphérique vient du fait que la place accordée au care/caring est considérée comme secondaire depuis la dernière partie du $\mathrm{XX}^{\mathrm{e}}$ siècle vis-à-vis du cure/curing : il s'agit là du soin technique qui guérit la maladie grâce aux progrès des sciences biomédicales (notamment par des molécules de synthèse aux effets thérapeutiques) et aux instruments issus des technologies récentes, dont l'imagerie médicale est un excellent exemple. Face à ce cure/curing, porté par la rationalité instrumentale et objective triomphante de la modernité, le soin classique qui se préoccupait à l'origine autant de prendre soin de l'âme des malades que de secourir leur corps a été marginalisé, rejeté d'une certaine manière dans une sphère moins noble, moins visible qui est la sphère du quotidien, des tâches profanes et domestiques: les aides-soignantes sont en charge des soins de nursing, qui constituent seulement la compétence 2 du référentiel de compétences infirmières qui compte dix compétences ${ }^{5}$. Cet objectif de guérir les maladies grâce aux progrès scientifiques appliqués au champ de la santé a permis d'indéniables progrès, mais il se heurte à une sorte de plafond de verre, à la fois pour des raisons financières et pour des raisons de disjonction dans leur rapport qui est plus segmenté que complémentaire avec le care/caring: la relation de soin est peu considérée, et ce faisant, la fonction de celles et ceux qui la mettent en place est faiblement reconnue. D'autre part, de nombreuses personnes ( 15 millions en France d'après le Haut Conseil de la santé publique $\left.{ }^{6}\right)$ souffrent de maladies chroniques qui impactent leur qualité de vie au quotidien sans pouvoir être l'objet d'une guérison en l'état actuel des connaissances. Le cloisonnement asymétrique entre cure/curing et care/caring constitue alors un frein à la prise en charge de ces patients, "soignés mais pas guéris ", pour reprendre l'expression de É. Rothier Bautzer (2016), qui mécaniquement relèvent davantage alors d'un caring inscrit dans un accompagnement sur une temporalité longue que d'un curing, ici inopérant, qui s'inscrit normalement dans un temps court (et appelé à l'être de plus en plus avec le développement de l'hospitalisation en ambulatoire). La question centrale, posée par Tronto, est celle de l'autonomie des patients. En visant à associer, comme le prône l'éducation thérapeutique, le patient chronique à la prise en charge des soins qui lui sont prodigués, on vise à maintenir ou développer son pouvoir d'agir en le rendant coopérateur de ces soins et en le responsabilisant, grâce à des médiations sur un temps long: le care/caring, seule voie possible pour réaliser cette tâche, retrouve alors une forme 
de légitimité. L'autonomie dont il est alors question n'est pas une autonomieindépendance qui est entravée par la maladie chronique mais une autonomieinterdépendance, en lien avec l'action des professionnels ou des aidants familiaux. Une recomposition possible des rapports entre cure/curing et care/caring se dessine, qui efface en partie une asymétrie de valeur intrinsèque, et promeut une nouvelle distribution des fonctions et des modalités d'action, qui, ensemble, visent l'autonomie. Dans la prise en charge de la population par le système de santé, le soin technique qui guérit les maladies (assez rapidement) se combinerait avec les soins apportés aux malades chroniques, aux personnes âgées dépendantes dont le poids démographique s'accroît, aux personnes handicapées dès l'école, et plus généralement aux personnes en situation de vulnérabilité, entravées pour un temps long dans leur pouvoir d'agir ordinaire. Le care/caring acquiert progressivement une nouvelle visibilité, marquée par des politiques inclusives qui ont pour fil directeur que la société ordinaire se soucie structurellement des personnes ayant des besoins particuliers : à l'école, dans les services et lieux publics et plus généralement dans la vie de la cité au quotidien.

\section{Bienveillance en éducation}

Depuis plus de trente ans, les travaux de Nel Noddings $(1984,2012)$ ont pour objet de problématiser la notion de caring dans le champ éducatif et scolaire, mais ils ont eu jusqu'à présent un impact moindre en France que les écrits dans le champ de la santé. On peut penser que la notion de bienveillance qui apparait dans la Loi de refondation de l'école (2013) fait écho à ces travaux et les importe dans le monde scolaire dans une dimension plus technique que politique: il s'agit bien ici d'infléchir des pratiques enseignantes dont les effets en matière d'attitude des élèves (décrochage scolaire important, symptômes de mal-être à l'école) et de résultats des élèves français, notamment dans les études PISA, sont en deçà des attentes.

\section{1 À l'origine, les travaux de Nel Noddings et leur lien avec les pédagogies actives}

Un point important que souligne Nel Noddings est que le caring ne recouvre pas seulement une dimension éthique, adossée à des dispositions innées proches de l'empathie, c'est-à-dire la capacité de se mettre à la place d'autrui, de ce qu'il ressent, mais aussi une dimension d'interdépendance et de réciprocité (Réto, 2016), qui ouvre sur des compétences communicationnelles et relationnelles, ici dans le champ éducatif. Ces dernières sont susceptibles d'être apprises et donc d'être l'objet d'un travail à dimension cognitive et réflexive, incluant, au-delà des intentions, des aspects techniques et, plus largement, des aspects liés à la relation pédagogique entre enseignants et élèves. Elle insiste notamment sur la valeur d'exemple de la posture et des pratiques de l'enseignant en classe en ce qui concerne le caring, sur la place qui doit être celle de la parole des élèves ou encore sur les pratiques coopératives en classe.

Il existe une convergence entre le caring en éducation (que l'on traduira par bienveillance éducative) et les idées et les pratiques des pédagogies nouvelles, dont les dix principes formalisés par François Chatelain ${ }^{7}$ donnent une idée synthétique pour ce qui concerne les objectifs, les attitudes et les processus pédagogiques. En cela, ils réactualisent la pensée de Coménius (1592-1670), l'un des fondateurs des pédagogies actives, qui proposait la 
métaphore célèbre du potier et du jardinier en éducation. D'un côté, le potier contraint l'argile qu'il façonne pour la faire advenir dans la forme qu'il veut lui donner: en éducation, c'est un modèle pédagogique ordonné à l'autorité magistrale qui énonce les règles, sanctionne le cas échéant et applique le programme scolaire ; d'un autre côté, le jardinier prend soin de la plante et cherche à favoriser son développement harmonieusement en répondant à ses besoins dans un contexte donné. À sa suite, de Joan Henrich Pestalozzi à Maria Montessori pour ce qui concerne les élèves à besoins éducatifs particuliers, de John Dewey pour la pédagogie du projet à Helen Parkhurst pour la pédagogie du contrat ou encore Célestin et Élise Freinet pour les pédagogies coopératives, nombre de pédagogues ont mis au centre de leur attention leur souci des enfants à travers la prise en compte de leurs besoins réels et du contexte concret des apprentissages ainsi que l'impérieuse nécessité d'accorder du crédit à leur parole, qui exprime leurs désirs, leurs difficultés et participe à les construire individuellement et collectivement. La proposition de la Loi pour la refondation de l'école vise à introduire un rééquilibrage entre ces deux modèles. En effet, le modèle du potier, ordonné au format scolaire dit traditionnel (Vincent, 1994) et porteur d'exigences fortes de l'école vis-à-vis des élèves, a été longtemps dominant. Il n'est pas, nous semble-t-il, obsolète mais semble, seul, moins pertinent dans un contexte scolaire marqué par la postmodernité et l'hétérogénéité des publics. Le modèle du jardinier, pour sa part, est resté largement aux périphéries du modèle dominant. Certes, petit à petit, certaines techniques et pratiques pédagogiques issues des pédagogies actives sont devenues poreuses au modèle traditionnel (classes de découverte, journal de classe, conseil d'élèves, projets pédagogiques...), mais l'esprit propre aux pédagogies actives est resté, peu ou prou, sur le seuil de l'école classique. Avec l'idée de bienveillance éducative, il s'agit de donner officiellement plus de visibilité et de centralité à des intentions et des pratiques pédagogiques qui manifestent un intérêt explicite au souci du sujet singulier qu'est chaque élève, à l'expression et la prise en charge de ses besoins effectifs, comme si l'école dans son ensemble devenait « ordinairement inclusive pour chacun ». Mais la Loi pour la refondation de l'école a le sens de la mesure: elle ne substitue pas la notion de bienveillance à la notion d'exigence, qui a fait des preuves. Elle vise, même si elle est peu diserte sur ce point, non pas à les juxtaposer ou les faire coexister mais à les mettre en synergie pour viser une meilleure réussite scolaire à la fois pour chaque élève et pour la société : un peu comme pour la marche, les deux jambes, la gauche et la droite, tiennent chacune leur rôle et ensemble, mises en rythme à bon escient, permettent de progresser vers une destination plus sûrement qu'en utilisant une seule des deux jambes. Ainsi, se rejoue, dans la tension dynamique entre exigence et bienveillance dans le champ éducatif, la relation de complémentarité entre cure et care que nous avons repérée dans le champ de la santé. La complexité et l'intensité des problèmes éducatifs et scolaires l'imposent comme une solution possible et raisonnable. Reste à passer des intentions aux pratiques, c'est-à-dire à mettre en mouvement le processus en quatre phases proposé par Joan Tronto. 


\subsection{Deux vecteurs au service de la tension entre exigence et bienveillance : le rythme des apprentissages et la place accordée aux espaces de dialogue en classe}

Pour donner une consistance à l'importance de la tension dynamique entre bienveillance et exigence dans le cadre scolaire, laquelle s'appuie sur une posture magistrale centrée sur les besoins de l'élève et sur des pratiques pédago-didactiques associées, nous mettons en avant deux paradigmes pédagogiques, certes déjà éprouvés, mais qui seraient à redécouvrir autrement qu'en étant réduits à des dispositifs désincarnés adressés à des flux d'élèves. Le premier est de porter une attention renouvelée à l'hétérogénéité des rythmes d'apprentissage dans la logique du pari d'éducabilité. Le second est de considérer comme centraux en pédagogie des espaces dialogiques, explicitement orientés vers les apprentissages, des espaces où questionner l'élève permettrait à l'enseignant de mener une enquête (Dewey, 1938/2006) sur l'activité cognitive de l'élève pour l'aider de manière ciblée en retour.

\section{Se soucier des rythmes d'apprentissage des élèves et apporter des médiations pédagogiques individualisées}

L'article très célèbre de Benjamin Bloom (1984) intitulé "Le défi des deux sigmas" suggère que, pour un enseignement donné à un groupe d'élèves, 90 \% d'entre eux peuvent réussir l'apprentissage visé sous réserve d'avoir le temps et de bénéficier de remédiation adaptées, à la fois sur le plan didactique et sur le plan pédagogique. Cependant, l'organisation des établissements scolaires est traditionnellement structurée autour de groupes de classes stables, qui, mécaniquement, vont avancer à une vitesse moyenne et ignorer l'hétérogénéité des rythmes d'apprentissage des élèves : dès le cours préparatoire de l'école élémentaire, cette hétérogénéité, à la fois inhérente à la variété des dispositions des élèves eux-mêmes et induite en partie par leur environnement social, culturel et matériel, mais aussi par les ressources et conditions d'apprentissage en classe ${ }^{8}$, est à la source de difficultés d'apprentissage. De son côté, Jérôme Bruner (1983) souligne combien un étayage individualisé, où l'enseignant a explicitement le souci d'apporter une aide à l'élève qui soit ajustée à sa démarche sur le plan cognitif et qui en même temps soit un soutien psychologique pour maintenir son engagement dans la résolution d'un problème, constitue une manière de prendre en charge les besoins spécifiques, dans leur singularité et à leur rythme. Une posture pédagogique à la fois exigeante et bienveillante, qui revient à mettre en place, au niveau des équipes enseignantes, des modalités concrètes d'organisation de l'offre d'enseignement qui permettent d'identifier dès leur apparition les difficultés d'apprentissage et de proposer des médiations ciblées. Les travaux de John B. Caroll (1963) autour de la pédagogie de la maîtrise (mastery learning), élargis par les travaux sur la régulation des apprentissages de Linda Allal (1988), ouvrent des pistes d'action qui sont aujourd'hui considérablement réactualisées par les perspectives d'individualisation induites par les outils numériques. Les différentes pistes pédagogiques, qu'a proposées dans le monde francophone le courant de la différenciation pédagogique (Perrenoud, 1992), vont également dans ce sens. Il s'agit alors de mobiliser conjointement les compétences didactiques et les compétences relationnelles et communicationnelles de l'enseignant pour promouvoir un processus d'enseignement qui 
serait juste (au sens de Tronto), ancré dans les réalités de la situation d'enseignementapprentissage et efficace.

\section{La place accordée aux espaces de dialogue en classe} précédent, insiste sur la qualité des interactions langagières maitre-élève au service des apprentissages et, par effet induit, sur le climat de la classe. Il s'agit de construire des espaces dialogiques de (re)médiations, dans le décours de la journée scolaire ou à l'occasion de dispositifs particuliers, et qui accordent un crédit solide à ce qu'a à dire un élève de son activité d'apprentissage et de son expérience d'apprenant, notamment lorsque cet apprentissage lui pose des difficultés. C'est une manière, certes basique, de prendre en compte ses besoins en tant qu'élève-acteur, mais c'est surtout une manière robuste pour identifier ce qui lui résiste, plutôt que de seulement constater une mauvaise performance dans la réalisation d'une tâche scolaire, sans passer par l'élève qui constitue pourtant une source d'information de première main. Car cela permet d'accéder à son expérience pratique d'élève, à la fois singulière et sensible, et à la difficulté sur le plan cognitif. Ce care about est souvent peu mis en évidence alors même qu'il conditionne l'ensemble du processus de taking care of et de care giving d'un point de vue didacticopédagogique.

L'enseignant-médiateur, comme le met en évidence Olivier Maulini (2005), doit s'efforcer de questionner l'élève plutôt que de l'interroger, même si cela est, pour lui, plus exigeant. Si le maître interroge l'élève et attend une réponse précise, il exerce une activité de contrôle et cela est utile mais insuffisant pour identifier la démarche de l'élève; en revanche, lorsque le maître questionne l'élève sur le processus d'apprentissage qu'il met en œuvre («Comment fais-tu pour trouver $4+6=9$ ?»), il lui demande d'expliciter une démarche mais ne connaît pas a priori ce qu'il va dire et qui est pourtant stratégique pour qu'une remédiation ajustée puisse l'aider à conscientiser et corriger son erreur. Le questionnement magistral ouvre un espace dialogique dédié à l'expression de la démarche cognitive de l'élève, qui, en même temps, est un espace qui reconnaît symboliquement et pratiquement l'élève comme un sujet singulier. Dans cette logique interactive, le maître, de son côté, a besoin de la confiance de l'élève pour faire son travail d'enseignant: on retrouve, embusquée, l'importance du climat de la classe et la nécessité de considérer que l'erreur est chose ordinaire lorsqu'on apprend. Ainsi, le maître met en œuvre, via ces espaces dialogiques, une forme de bienveillance centrée sur l'activité d'apprentissage de l'élève. Cette bienveillance pédagogique de l'enseignant participe à sécuriser la prise de risque et à valoriser l'effort que constitue l'activité d'apprentissage de l'élève, suscitant un maintien de l'engagement de ce dernier dans la tâche scolaire. Les espaces dialogiques, bien loin de saper l'autorité magistrale, s'entendent comme une forme d'exigence, donnant lieu à un contrat plus ou moins explicite pour que l'élève ne réitère pas l'erreur corrigée et se maintienne sur la voie de la réussite scolaire. La fonction qu'exercent ces espaces dialogiques est tout à fait en synergie avec le courant de la pédagogie explicite (Bonnery, 2007, Gauthier, Bissonnette \& Richard, 2013) qui vise à redonner plus de sens à l'activité scolaire des élèves, s'appuyant clairement sur la bienveillance magistrale à travers le repérage de leurs besoins et l'ajustement des réponses didactiques et pédagogiques. 


\section{Conclusion : la bienveillance, une priorité encore fragile}

Le courant critique nord-américain, dont Joan Tronto est une figure importante, a, dès les années 1970, participé à conceptualiser la notion de care/caring, qui suggère de prendre en considération le souci d'autrui, notamment un autrui vulnérable, à travers la prise en compte et la prise en charge de ses besoins concrets. Ce courant met en avant l'expérience ordinaire des personnes qui offrent et des personnes qui bénéficient de la bienveillance et met en exergue l'interdépendance entre les acteurs Le monde de la santé, à travers les notions antagonistes et complémentaires de cure/curing et de care/caring, s'est emparé de cette question de l'attention à autrui et de la sollicitude, et s'efforce, non sans difficultés, de lui donner corps sur les plans épistémologique et pratique. Plus récemment, sous la traduction de bienveillance éducative, la notion de caring s'invite dans le registre scolaire, tout en restant assez floue sur le plan épistémologique. La prise en compte plus affirmée des rythmes d'apprentissage de chacun ainsi que des médiations plus ciblées, notamment appuyées sur des interactions langagières entre enseignant et élèves, seraient deux leviers pour donner une consistance pédagogique et didactique à cette notion de "bienveillance éducative» et opérationnaliser les intentions qu'elle suggère. Sans doute faudrait-il pour cela que les équipes éducatives elles-mêmes, mais aussi la formation des enseignants, la prennent en charge pour éviter de tomber dans une simple mode pédagogique qui ne serait que de passage.

\section{BIBLIOGRAPHIE}

Allal, L. (1988). Vers un élargissement de la pédagogie de la maîtrise : processus de régulation interactive, rétroactive, proactive. Dans M. Huberman (dir.), Assurer la réussite des apprentissages scolaires : les propositions de la pédagogie de la maîtrise (pp. 86-126). Neufchâtel : Delachaux et Niestlé.

Bloom, B. (1984). « The 2 sigmas problem ». Educational Researcher, 13, 4-16.

Bonnery, S. (2007). Comprendre l'échec scolaire. Élèves en difficultés et dispositifs pédagogiques. Paris : La Dispute.

Bruner, J. S. (1983). Savoir faire, savoir dire, le développement de l'enfant. Paris : PUF.

Bucheton, D. \& Soulé, Y. (2009). Les gestes professionnels et le jeu des postures de l'enseignant dans la classe : un multi-agenda de préoccupations enchâssées. Éducation et didactique, 3(3).

Carroll, J. B (1963). Un modèle d'apprentissage scolaire. Teachers College Record, 64, 723-733.

Dewey, J. (2006). Logique : la théorie de l'enquête (1938). Paris : PUF.

Fisher, B. \& Tronto, J. (1991). Toward a feminist theory of caring. Dans E. Abel \& M. Nelsin (dir.), Circles of care: Work and Identity in Women's Lives (pp. 36-54). Albany, NY : State University of New York Press. 
Gauthier, C., Bissonnette, S. \& Richard, M. (2013). Enseignement explicite et réussite des élèves. La gestion des apprentissages. Paris : De Boeck.

Gilligan, C. (1982). In a Different Voice. Psychological theory and women. Cambridge : Harvard University Press.

Hesbeen, W. (2014). Pour une éthique du quotidien des soins. Cancer(s) et psy(s), 1, 173-177.

Laugier, S. \& Paperman, P. (dir.) (2006). Le Souci des autres. Éthique et politique du care. Paris : EHESS. Maulini, O. (2005). Questionner pour enseigner et pour apprendre : le rapport au savoir dans la classe. Paris : ESF.

Mauss, M. (2013). Essai sur le don (1924). Paris : La République des Lettres.

Noddings, N. (1984). Caring, a Feminine Approach to Ethics and Moral Education. Berkeley : University of California Press.

Nodding, N. (2012). The caring relation in teaching. Oxford Review of Education, XXXVIII(6), 771-781. Perrenoud, P. (1992). Différenciation de l'enseignement : résistances, deuils et paradoxes. Cahiers pédagogiques, 306, 49-55.

Piot, T. (2008). La construction des compétences pour enseigner. Mc Gill Journal of Education (Montréal, Canada), 43(2), 95-110.

Piot, T. (2017). L'émancipation : un lien génétique et ambivalent avec les sciences de l'éducation. Dans J.-F. Marcel \& D. Broussal (dir.), Émancipation et recherche en éducation (pp. 355-375). Toulouse : Éditions du Croquant.

Rawls, J. (1997). Théorie de la justice (1971). Paris : Le Seuil.

Réto, G. (2016). Le caring, une voie pour reconfigurer l'École française au moment de sa refondation? Éducation et socialisation, 40. Repéré à http://edso.revues.org/1504.

Rothier Bautzer, É. (2013). Le care négligé. Les professions de santé face au malade chronique. Paris : De Boeck ESTEM.

Rothier Bautzer, É. (2016). Dictionnaire des concepts en soins infirmiers. Vocabulaire dynamique de la relation soignant-soigné (Rubrique « caring/care », pp. 75-80 et rubrique « curing/cure », pp. 80-81; C. Paillard coord.). Paris : SETES Édition.

Solomon, B. (1976). Black Empowerment: Social Work in Oppressed Communities. New York : Columbia University Press.

Tronto, J. (2009). Un monde vulnérable. Pour une politique du care. Paris : Éditions La Découverte.

Vincent, G. (dir.) (1994). L'éducation prisonnière de la forme scolaire? Scolarisation et socialisation dans les sociétés industrielles. Lyon: Presses universitaires de Lyon.

Wise, J. B. (2005). Empowerment Practice with Families in Distress. New York : Columbia University Press.

\section{NOTES}

1. Loi $n^{\circ} 2013-595$ du 8 juillet 2013 d'orientation et de programmation pour la refondation de l'École de la République.

2. Circulaire $\mathrm{n}^{\circ}$ 2014-068 du 20 mai 2014 (NOR : MENE1411580C). 
3. Le terme care renvoie plus à un objet, le soin adressé à l'autre et le terme caring au processus pratique associé.

4. Liane Mozère a rédigé un avant-propos engagé de la traduction en français de l'ouvrage de Joan Tronto (2009).

5. Profession Infirmier (2015). Recueil des principaux textes relatifs à la formation préparant au diplôme d'État et à l'exercice de la profession. (Réf 531 200). Paris : Berger-Levrault.

6. http://www.hcsp.fr/explore.cgi/Adsp?clef=112.

7. https://www.ecolelasource.org/UserFiles/file/Pedagogie/ Les_10_Principes_de_lEducation_Nouv.pdf.

8. Dominique Bucheton et Yves Soulé (2009) rendent compte de ces ressources et conditions d'apprentissage dans un schéma très opératoire.

\section{RÉSUMÉS}

Le couple «bienveillance-exigence» est une notion centrale de la loi. Sur un plan épistémologique mais aussi un plan pratique, la notion de bienveillance se rattache à la notion de caring issue d'un courant de pensée alternatif né aux USA dans les années 1980 et qui s'intéresse aux personnes en situation de vulnérabilité. Dans un premier temps, nous présentons cette notion de caring à travers son usage dans les milieux du soin. Mais on peut considérer, avec Noddings, que, d'une certaine manière, les enfants qui rencontrent des difficultés scolaires sont aussi des personnes vulnérables. Nous proposons de formaliser un lien entre la bienveillance en éducation et les pédagogies actives, notamment à partir de deux vecteurs : la prise en compte du rythme des apprentissages et la place accordée à des espaces de dialogue en classe. Cependant, il semble que la notion de bienveillance demeure encore une priorité fragile en classe.

The union "benevolence-requirement" is a central notion of the law. On an epistemological level but also a practical level, the notion of benevolence is related to the concept of caring resulting from an alternative way of thinking born in the USA in the years 1980 and which focuses on people in vulnerable situations. At first, we present this concept of caring through its use in care environments. But we can consider, with Noddings, that in a certain way children who have educational difficulties are also vulnerable people. We suggest to formalize a link between benevolence in education and active pedagogies, notably from two vectors: taking into account the rhythm of learning and the space given to allow use of dialogue in the classroom. However, it seems that the notion of benevolence still remains a fragile priority in the classroom.

\section{INDEX}

Mots-clés : bienveillance, caring, pédagogies actives, rythmes d'apprentissage, dialogue

Keywords : benevolence, caring, active pedagogies, dialogue learning rythm 


\section{AUTEUR}

\section{THIERRY PIOT}

Professeur en sciences de l'éducation à l'université de Caen Normandie et dirige le laboratoire normand CIRNEF EA 7454. Ses travaux portent sur le développement professionnel dans les métiers de services adressés à autrui, et notamment sur les fonctions des interactions langagières comme ressource dans ces métiers. 